\title{
SHAPE OSCILLATIONS OF A PARTICLE COATED BUBBLE DURING RISE IN A LIQUID COLUMN
}

\author{
Prithvi R.Y \\ Department of Metallurgical and Materials Engineering \\ Indian Institute of Technology Madras \\ Chennai, India
}

\author{
Sabita Sarkar \\ Department of Metallurgical and Materials Engineering \\ Indian Institute of Technology Madras \\ Chennai, India \\ sabita.sarkar@iitm.ac.in
}

\begin{abstract}
Particle coated bubble and its stability plays a major role during particle recovery in flotation process. $A$ rising bubble undergoes shape oscillations which are subjected to change when particles are coated on the surface of a bubble. Experiments were performed to understand the effect of particle coating on a rising bubble in a liquid column. Hydrophobic Low density polyethylene particles were used to coat the bubble surface and water was used as liquid medium. Two images (one direct and mirror image) were taken for all position during rise of the bubble. Effect of different fraction of particle coating on the bubble surface oscillations was studied. It is observed that the shape oscillations of bubbles are arrested as coating fraction increased from $10 \%$ to $50 \%$ with the latter undergoing almost no deformation in shape. The bubble in this case behaves like a rigid body and exhibits pure rotation as it moves up.
\end{abstract}

Keywords-shape oscillations; particle coated bubble; single bubble rise; coating fraction.

\section{INTRODUCTION}

Bubble flotation in presence of particles is a phenomena which has wide spread application like waste water treatment, petrochemical plants, froth flotation process, paper industry and in refining operations like secondary steel making process. Understanding the behaviour of bubble motion and its characteristics in presence of particles is a key step in evaluating preliminary variables like air flow rate, bubble size, particle size etc. with respect to its application in each field. A significant amount of research has been carried out in understanding the behaviour of a single bare bubble rising in a fluid column $[2,6]$. The theory behind shape oscillations of a single rising bubble has been given in detail by several researchers [1,3 \& 5]. However motion of particle coated bubble in liquid medium is not so evident in literature. In this work, motion of particle coated single bubble was studied experimentally. The effect of particle coating on the bubble surface oscillation and the overall bubbly motion was main focus of this study. Strongly hydrophobic polymeric Low Density Polyethylene (LDPE) particles were chosen with interest to alumina inclusions present in molten steel [4]. The shapes of bubbles were chosen in the ellipsoidal regime which is commonly used for removal of alumina inclusion in tundish [7].

\section{EXPERIMENTAL METHODOLOGY}

In order to study the shape oscillations of single bubble during rise with particles coated on the bubble surface, a bubble column made of plexi-glass was fabricated. A schematic representation of the experimental set-up is shown in Figure 1. The tank comprised of two compartments located one below the other. Initially the tank was filled with distilled water up to a height of $0.55 \mathrm{~m}$ in the presence of polymeric LDPE particles inside the lower compartment. The particles which have specific gravity of 0.92 were prevented from floating to the upper compartment by providing a slide door whose opening was controlled manually. An air bubble was held at the tip of a nozzle using an infusion pump and the particlewater mixture was stirred inside the compartment with the help of an impeller arrangement powered by a universal motor. The speed of rotation of the impeller and duration of rotation controlled the coating fraction of particles on the bubble surface. In this study the size of LDPE particles chosen were from 150-210 microns and the bubble sizes were 6.8 and $4.2 \mathrm{~mm}$. After allowing sufficient time for the particle-water mixture to reach a quiescent state the slide door was opened and the air pump was operated again. The particle laden bubble was detached from the nozzle and moved upwards due to buoyancy.

A test section between the heights $0.019 \mathrm{~m}$ to $0.028 \mathrm{~m}$ in the upper portion of the tank was illuminated with white coloured diffuse back lighting. This was the region where the particle coated bubble behaviour was studied. A mirror was placed inside the tank at an angle of $45^{\circ}$ to the camera axis. Light was projected on to the mirror using a screen as a reflector and this method provided back lighting for front view where the bubble appeared white with dark background and the side (reflected) lighting illuminated the mirror image. In this way both the front and side views of the bubble could be obtained using a single camera placed in front of the experimental setup. 


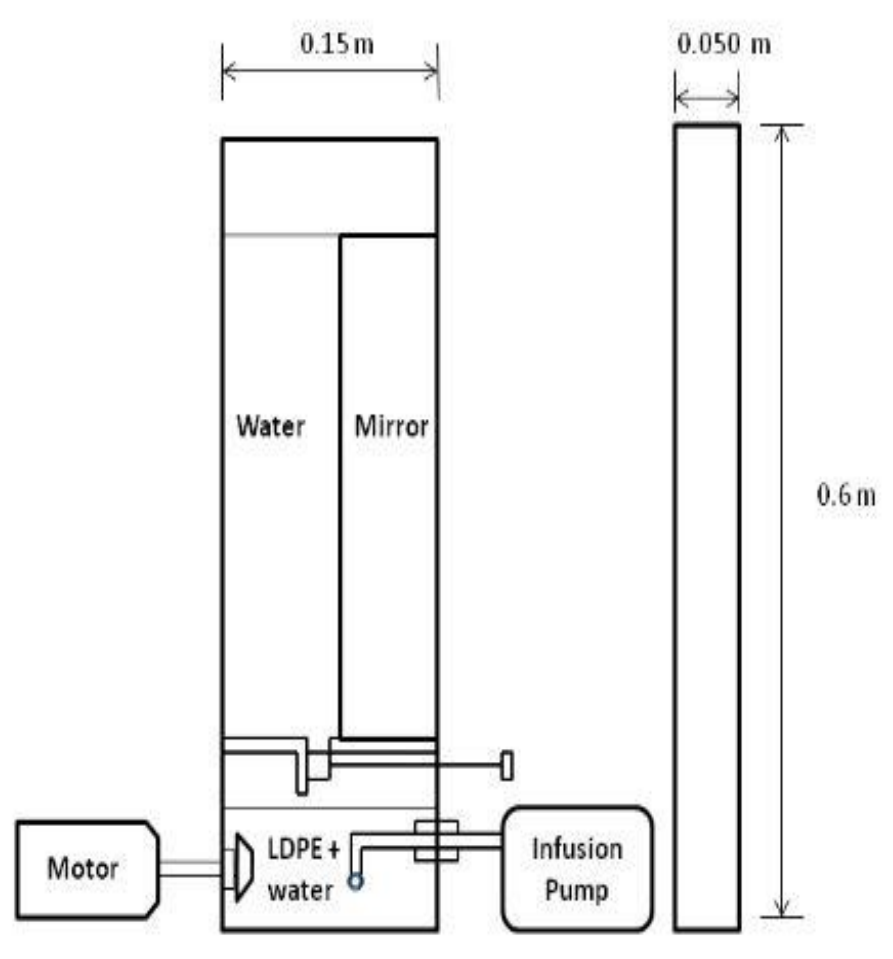

Figure 1. Schematic of the Experimental Set-up

The bubble rise and shape oscillations was captured using a CMOS camera (Teledyne Dalsa) having a frame rate of 300 frames per second and a resolution of $640 \times 480$ pixels. The images recorded were originally in greyscale format.

The greyscale images were then converted to binary format in subsequent steps by the method of intensity thresholding, using Matlab-14 software. At these stage properties like major axes, minor axes, centroid, and bubble boundary were measured using Matlab 14 - image processing toolbox. In order to determine the particle coating fraction on the bubble, the area of particle coverage was determined by fitting a closed spline around the periphery of the region covered with particles using Image J-image processing software.

\section{RESULTS AND DISCUSSION}

\section{A. Behaviour of Particle coated Bubble}

Bubbles with different extent of particle coating and at different times were observed during the experiments. In case of the bubble with $10 \%$ particle coating at the surface, oscillations were altered and reduced drastically from the case of that of bare bubble. The oscillations were accompanied by rotation of the bubble as it moved upwards. The region where the interface was coated with particles showed no surface oscillation and behaved like solid surface. The surface oscillations were prevalent only at the regions where there were no particles. This altered the overall oscillating behaviour of the bubble.

When the extent of coating on the bubble surface increased to almost half of the bubble surface area, the oscillations were completely arrested. The polymer particles completely retarded the surface deformation and the bubble did not undergo any shape oscillation during rise. Instead the bubble exhibited only rotation about its minor axis. The flow past the bubble at the boundary experienced no slip at regions where the surface retardation had occurred. Visual images of a bubble of $6.8 \mathrm{~mm}$ diameter with different particle coatings and at different times are compared and shown in Figure 2. The particle coating on the bubble increases drag force that it experiences and reduces the rise velocity.

Experiments were done with a bubble having diameter of 4.2 $\mathrm{mm}$ (Figure 3) and a similar behaviour was observed. The only difference was that the amplitude of oscillations undergone by the $4.2 \mathrm{~mm}$ bubble was comparatively lesser than that of the $6.8 \mathrm{~mm}$ bubble. The rise velocity in this case is more when compared to that of $6.8 \mathrm{~mm}$ diameter bubble, as coating fraction is more and the bubble area is lesser. The force due to buoyancy for the $4.2 \mathrm{~mm}$ bubble exceeds the drag force.

\section{B. Effect of particle coating on shape oscillations}

The shape oscillations of bubbles are generally expressed in terms of spherical harmonics with 2,0 and 2,2 as the dominant modes [3]. These two modes are the dominant modes of shape oscillation for an ellipsoidal bubble. Mode 2,2 shape oscillations of the bubble are axisymmetric in nature. In this mode, the capillary wave is assumed to travel around the equator of the bubble and is characterized by the ratio of major axes obtained from the direct image $\left(d_{d}\right)$ to the major axis of that from the mirror image $\left(d_{m}\right), R=d_{d} / d_{m}$. The interpretation of this mode of oscillation is that the bubble is an ellipsoid rotating about its minor axis as it travels vertically upwards and as it does a 2-D projection of the 3-D bubble in either the front or side plane will constitute major axes of different lengths. Thus the ratio of major axes $\mathrm{R}$ interprets change in the major axes length due to rotation about its minor axes.

Figure 4 shows mode 2,2 oscillations for bare bubble and $10 \%$ particle coated bubble. The $6.8 \mathrm{~mm}$ bubble shows a distinctive difference in oscillation between the bare bubble and a particle coated bubble, whose values of $\mathrm{R}$ are varying from 0.5 to 1.5 for a bare bubble and limited from 0.8 to 1.2 for the particle coated bubble. This is an indication that the particle coating has a decreasing effect on the extent of elongation of the major axes. However for the $4.2 \mathrm{~mm}$ bubble size, particle coating seems to have resulted in increased 


\section{ELK}

\section{Asia Pacific Journals}

length of one of the major axis (one seen from the direct image) as the value of $R$ remains always greater than unity.

Mode 2,0 shape oscillation is represented by equivalent major axis defined as $d_{e q}=\sqrt{ }\left(d_{d} \times d_{m}\right)$. This is an interpretation of the bubble fluctuating from oblate spheroid to

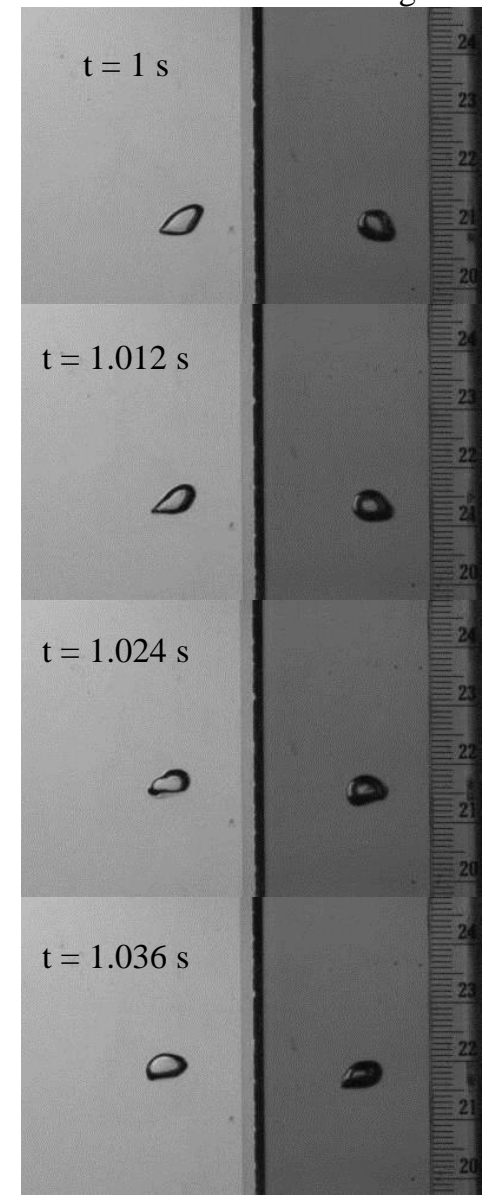

A

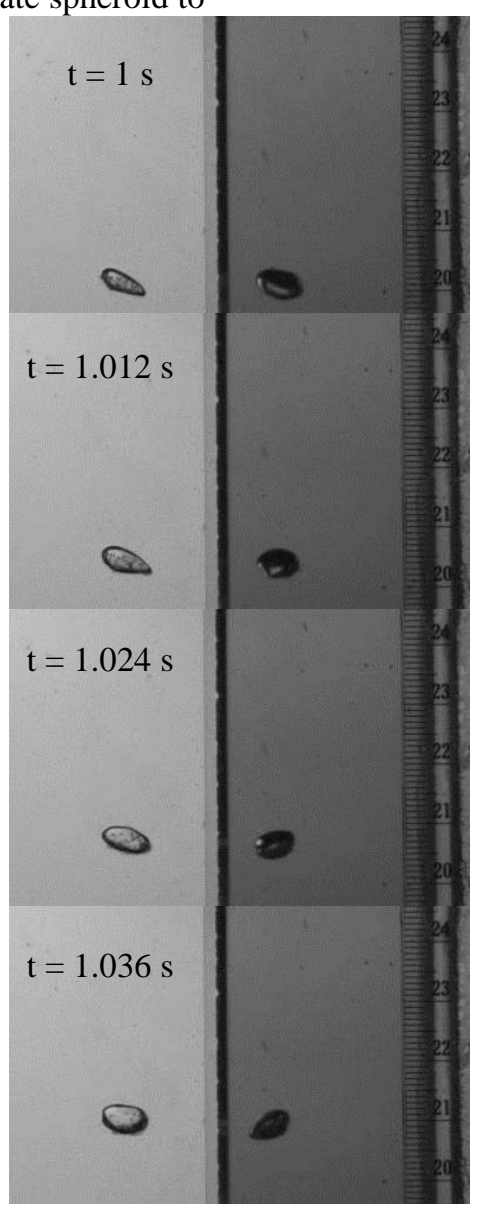

B prolate shape. In reality it does not transform completely to prolate shape but as it tends to oscillate in the 2,0 mode with alternating elongation and contraction of the major axes. This mode of oscillation is non-axisymmetric in nature and the

Figure 2. Images of a $6.8 \mathrm{~mm}$ bubble for a time up to $0.036 \mathrm{~s}$ A) bare bubble undergoing shape deformation B) $10 \%$ coated bubble undergoing partial deformation C) $50 \%$ coated bubble with no deformation
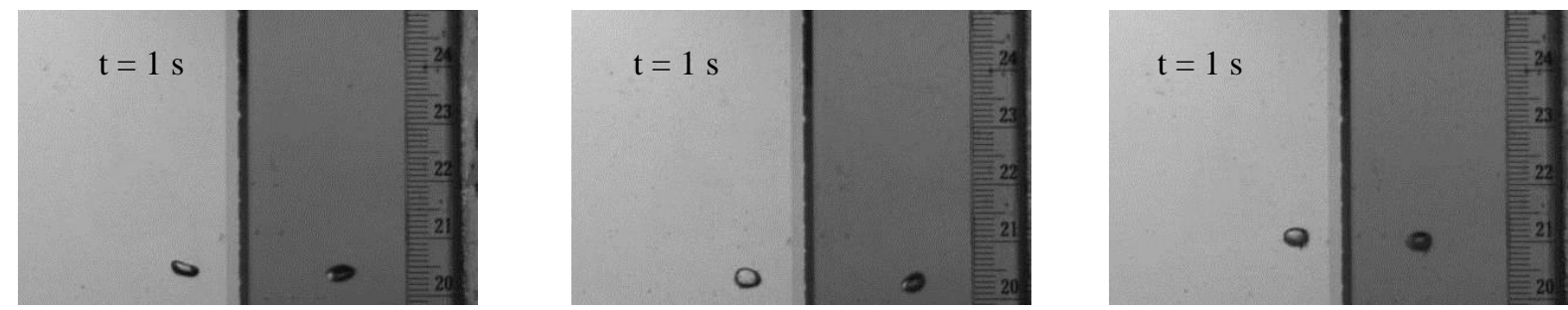
ELK

Asia Pacific Journals

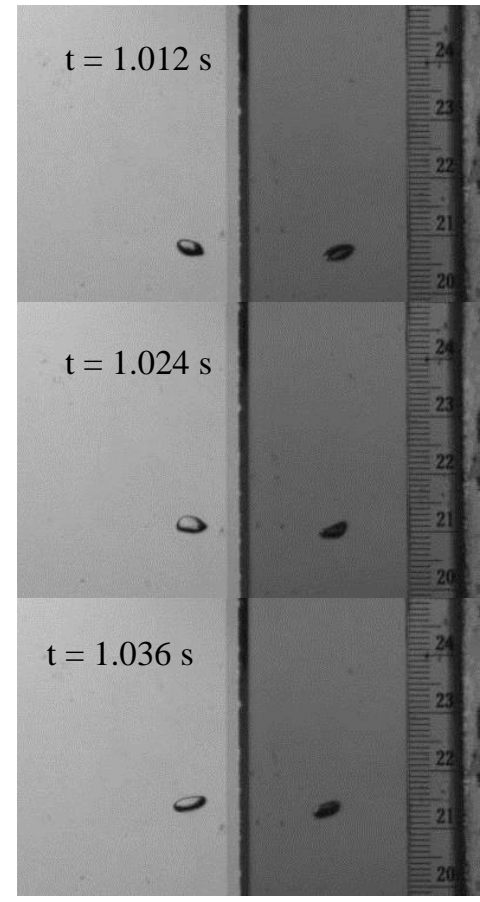

A

ELK Asia Pacific Journals - Special Issue

ISBN: 978-81-930411-4-7

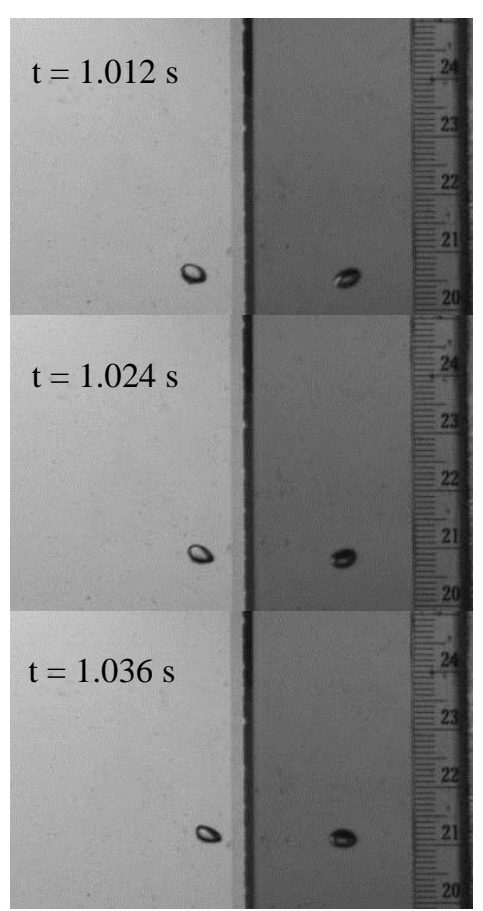

B

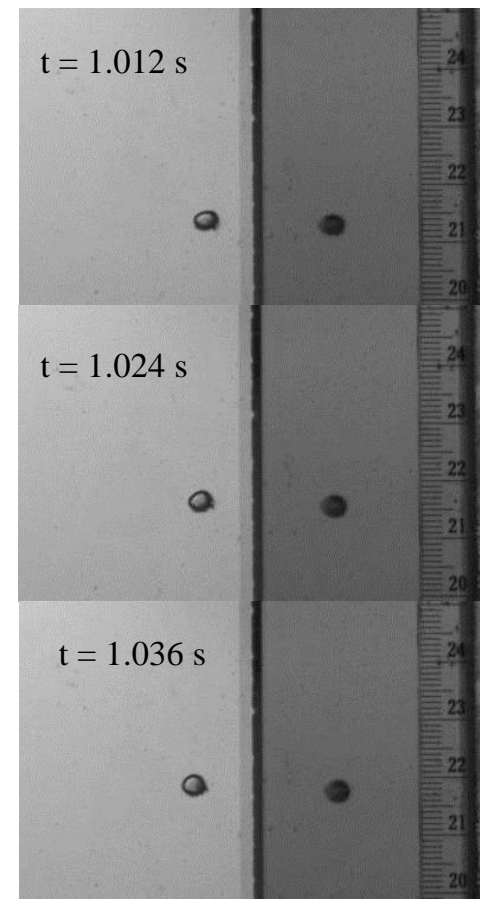

C

Figure 3. Images of a $4.2 \mathrm{~mm}$ bubble for time up to $0.036 \mathrm{~s} \mathrm{A)} \mathrm{bare} \mathrm{bubble} \mathrm{undergoing} \mathrm{shape} \mathrm{deformation} \mathrm{B)} 10 \%$ coated bubble undergoing partial deformation C) $50 \%$ coated bubble with no deformation

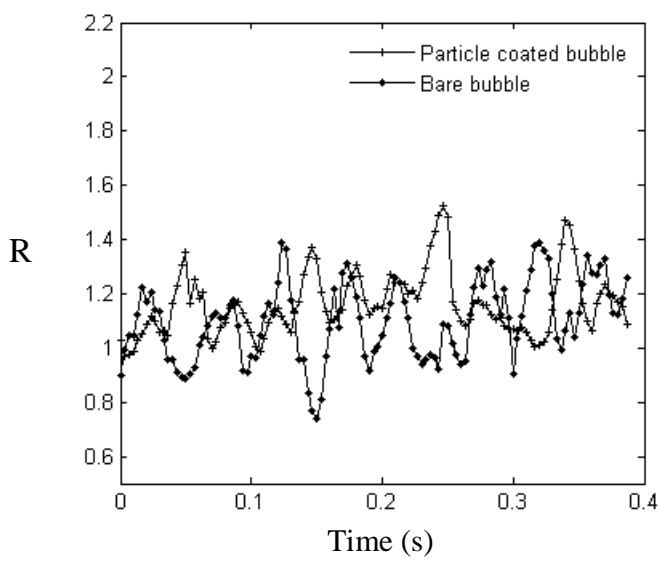

A

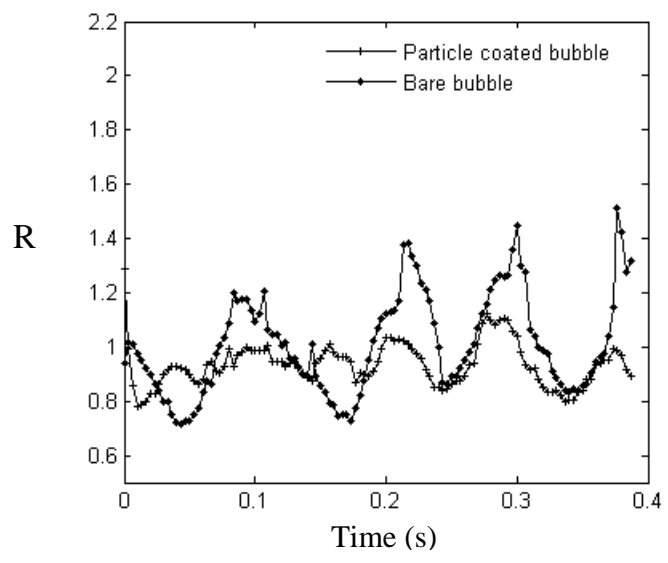

B

Figure 4. Plot of $\mathrm{R}$ vs time for bare bubble and $10 \%$ particle coated bubble. A) $4.2 \mathrm{~mm}$ bubble B) $6.8 \mathrm{~mm}$ bubble

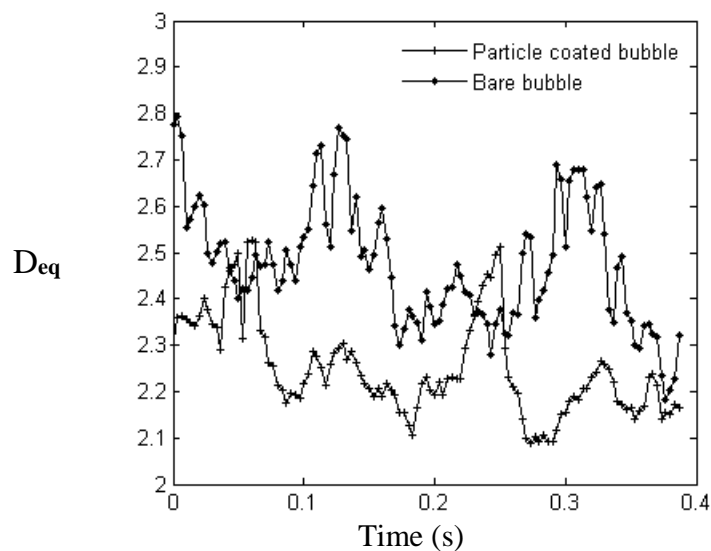

A

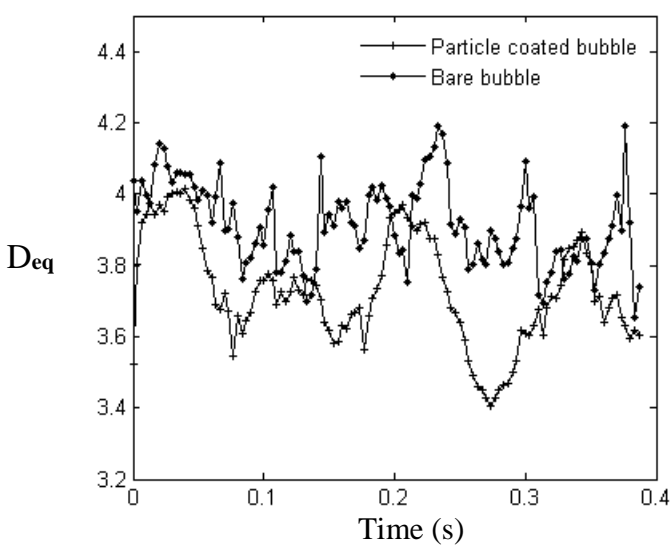

B 
Figure 5. Plot of $D_{\text {eq }}$ vs time for bare bubble and $10 \%$ particle coated bubble. A) $4.2 \mathrm{~mm}$ bubble B) $6.8 \mathrm{~mm}$ bubble Physics of fluids, vol. 11(7), pp. 1781-1796, 1999.

capillary waves are assumed to be moving around the bubble from one end of the bubble pole to another. Thus it is characterized by obtaining an equivalent diameter of a circle whose surface area is the same as that of the ellipse in the cross-section plane containing the two major axes $\left(D_{e q}\right)$.

It can be seen from Figure 5 that in the case of a bare bubble as well as partially coated bubble the amplitude of 2, 0 mode of oscillation is greater for the size corresponding to $6.8 \mathrm{~mm}$ bubble size since it naturally has greater major axes lengths. For this bubble size when particles are coated the elongation in major axis length gets reduced since the particles inhibit wave action at the bubble surface and behave as a rigid body. For the $4.2 \mathrm{~mm}$ bubble size the arrest in major axes shrinkage is seen more predominantly as there is a drastic decrease in amplitude of $\mathrm{D}_{\mathrm{eq}}$ of particle coated bubble. The complete prevention of surface deformation when almost half of the bubble surface area was coated can be evidently seen in the images in Figure 2C and Figure 3C and it is inappropriate to

use surface harmonics to describe such a body which does not undergo shape oscillation. As the bubble surface deformation is completely arrested the restoring capillary force is retarded preventing any further shape oscillation.

\section{CONCLUSIONS}

- LDPE particles do not detach from the bubbles for both sizes of $4.2 \mathrm{~mm}$ and $6.8 \mathrm{~mm}$ due to shape oscillations during rise in the static liquid column.

- When the coating fraction on the bubbles is as mild as $10 \%$, the bubble surface deformation occurs only at regions, where particles are absent. Thus overall oscillation in amplitude is reduced

- The reduction in elongation of major axes is more for a particle coated smaller ellipsoidal $(4.2 \mathrm{~mm}$ size $)$ than the larger ellipsoidal bubbles $(6.8 \mathrm{~mm}$ size $)$.

- Heavily coated bubbles with coating fraction as high as $50 \%$, shows no deformation behaviour, instead they behaves like a rigid body undergoing pure rotation during rise.

\section{REFERENCES}

[1] C. Brucker, "Structure and Dynamics of the wake of the bubbles and its relevance for bubble interaction,"
[2] P.C. Duineveld, “ The rise velocity and shape of bubbles in pure water at high Reynolds number," J.Fluid Mech, vol 292, pp. 325-332, 1995.

[3] K. Lunde, R.J. Perkins, "Shape Oscillations of Rising Bubbles," Applied Scientific Research, vol.58, pp.387-408, 1998

[4] J.P. Rogler, "Modeling of inclusion removal in a tundish by gas bubbling," M.S. Thesis, Ryerson University, 2001.

[5] C. Veldhuis, A. Biesheuvel , L. van Wijngaarden, "Shape oscillations on bubbles rising in clean and in tap water," Physics Of Fluids, vol.20, pp. 1-12, 2008.

[6] A.W.G de Vries, A. Biesheuvel, L. van Wijngaarden, "Notes on the path and wake of a gas bubble rising in pure water," International Journal of Multiphase Flow, vol.28(11), pp.1823-1835, 2002.

[7] L.Zhang, S.Taniguchi, "Fundamentals of inclusion removal from liquid steel by bubble Flotation," International Materials Reviews, vol. 45(2), pp.59-82, 2000 . 